\title{
A New Extension of Humbert Matrix Function and Their Properties
}

\author{
Ayman Shehata, Mohamed Abul-Dahab* \\ ${ }^{1}$ Department of Mathematics, Faculty of Science, Assiut University, Assiut, Egypt \\ ${ }^{2}$ Department of Mathematics, Faculty of Science, South Valley University, Qena, Egypt \\ E-mail: "mamabuldahab@yahoo.com \\ Received August 15, 2011; revised October 22, 2011; accepted October 30, 2011
}

\begin{abstract}
This paper deals with the study of the composite Humbert matrix function with matrix arguments $J_{A, B}(\underline{z})$. The convergence and integral form this function is established. An operational relation between a Humbert matrix function and Kummer matrix function is studied. Also, integral expressions of this relation are deduced. Finally, we define and study of the composite Humbert Kummer matrix functions.
\end{abstract}

Keywords: Hypergeometric Matrix function, Humbert Matrix Function, Kummer Matrix Function, Integral Representations

\section{Introduction}

Special matrix functions appear in the literature related to statistics [1-4] and more recently in connection with matrix analogues of Laguerre, Hermite and Legendre differential equations and the corresponding polynomial families [5-7]. The connection between the Humbert matrix function and modified Bessel matrix function has been established in $[8,9]$. In recent papers $[10,11]$, we defined and studied the Humbert matrix functions. The Kummer's confluent hypergeometric function belongs to an important class of special functions of the mathematical physics with a large number of applications in different branches of the quantum mechanics atomic physics, quantum theory, nuclear physics, quantum electronics, elasticity theory, acoustics, theory of oscillating strings, hydrodynamics, random walk theory, optics, wave theory, fiber optics, electromagnetic field theory, plasma physics, the theory of probability and the mathematical statistics, the pure and applied mathematics in $[3,4,12-14]$. Recently, an extension to the Kummer matrix function of complex variable is appeared in [15]. The first author has earlier studied the certain Kummer matrix function of two complex variables under certain differential and integral operators [16]. The primary goal of this paper is to consider a new system of matrix functions, namely the composite Humbert matrix function, Humbert Kummer matrix function and composite
Humbert Kummer matrix function.

The paper is organized as follows: Section 2 is define and study of the composite Humbert matrix function. The convergence and integral form is established. In Section 3 an operational relation between a Humbert matrix function and Kummer matrix function is given. Integral expressions of Humbert Kummer matrix functions are deduced. In Section 4 we defined and studied of the composite Humbert Kummer matrix functions.

Throughout this paper $D_{0}$ will denote the complex plane. A matrix $P$ is a positive stable matrix in $C^{N \times N}$ if $\operatorname{Re}(\lambda)>0$ for all $\lambda \in \sigma(P)$ where $\sigma(P)$ is the set of all eigenvalues of $P$ and its two-norm denoted by

$$
\|P\|=\sup _{x \neq 0} \frac{\|P x\|_{2}}{\|x\|_{2}},
$$

where for a vector $y$ in $C^{N},\|y\|_{2}=\left(y^{T} y\right)^{\frac{1}{2}}$ is the Euclidean norm of $y$.

Let $\alpha(P)$ and $\gamma(P)$ be the real numbers which were defined in [17] by

$$
\begin{aligned}
& \alpha(P)=\max \{\operatorname{Re}(z): z \in \sigma(P)\}, \\
& \gamma(P)=\min \{\operatorname{Re}(z): z \in \sigma(P)\} .
\end{aligned}
$$

If $f(z)$ and $g(z)$ are holomorphic functions of the complex variable $z$ which are defined in an open set $\Omega$ of the complex plane and $P$ is a matrix in 
$C^{N \times N}$ such that $\sigma(P) \subset \Omega$, then from the properties of the matrix functional calculus [18], it follows that

$$
f(P) g(P)=g(P) f(P) .
$$

Hence, if $Q$ in $C^{N \times N}$ is a matrix for which $\sigma(Q) \subset \Omega$ and if $P Q=Q P$, then

$$
f(P) g(Q)=g(Q) f(P) \text {. }
$$

The reciprocal Gamma function denoted by $\Gamma^{-1}(z)=\frac{1}{\Gamma(z)}$ is an entire function of the complex variable $Z$. Then the image of $\Gamma^{-1}(z)$ acting on $P$ denoted by $\Gamma^{-1}(P)$ is a well-defined matrix. Furthermore, if

$$
P+n I \quad \text { is invertible for all integer } n \geq 0 .
$$

The Pochhammer symbol or shifted factorial defined [17] by

$$
\begin{aligned}
(P)_{n} & =P(P+I) \cdots(P+(n-1) I) \\
& =\Gamma(P+n I) \Gamma^{-1}(P) ; \quad n \geq 1 ;(P)_{0}=I
\end{aligned}
$$

Jódar and Cortés have proved in [17] that

$$
\Gamma(P)=\lim _{n \rightarrow \infty}(n-1) !\left[(P)_{n}\right]^{-1} n^{P} .
$$

Let $P$ and $Q$ be two positive stable matrices in $C^{N \times N}$. The gamma matrix function $\Gamma(P)$ and the beta matrix function $B(P, Q)$ have been defined in [19] as follows

$$
\begin{aligned}
& \Gamma(P)=\int_{0}^{\infty} \mathrm{e}^{-t} t^{P-I} \mathrm{~d} t \\
& t^{P-I}=\exp ((P-I) \ln t)
\end{aligned}
$$

and

$$
B(P, Q)=\int_{0}^{1} t^{P-I}(1-t)^{Q-I} \mathrm{~d} t .
$$

The Schur decomposition of $P$, was given by [18] in the form:

$$
\left\|\mathrm{e}^{t P}\right\| \leq \mathrm{e}^{t \alpha(P)} \sum_{s=0}^{r-1} \frac{\left(\|P\| r^{\frac{1}{2}} t\right)^{s}}{s !} ; \quad t \geq 0,
$$

and

$$
\begin{aligned}
& \left\|n^{P}\right\| \leq n^{\alpha(P)} \sum_{s=0}^{r-1} \frac{\left(\|P\| r^{\frac{1}{2}} \ln n\right)^{s}}{s !} ; n \geq 1 .
\end{aligned}
$$

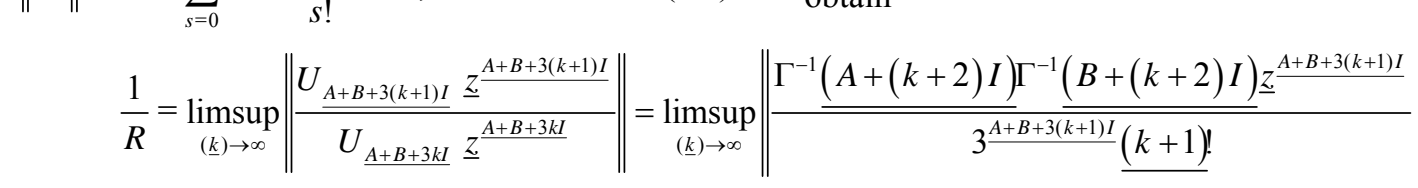

$$
\begin{aligned}
& \div \frac{\Gamma^{-1}(\underline{A+k I}) \Gamma^{-1}(\underline{B+k I}) \underline{z}^{\underline{A+B+3 k I}}}{3^{\frac{A+B+3 k I}{k}} \underline{!}}\left\|\leq \limsup _{(\underline{k}) \rightarrow \infty}\right\| \frac{(A+(k+1) I)^{-1}(B+(k+1) I)^{-1} \underline{z}^{3}}{\underline{k+1}} \|\left|\underline{z}^{3}\right|
\end{aligned}
$$

\section{Composite Humbert Matrix Function}

Let us introduce the following notation (see [20])

$$
\begin{aligned}
& \underline{k}=\left(k_{1}, k_{2}, \cdots, k_{s}\right), \\
& (\underline{k})=k_{1}+k_{2}+\cdots+k_{s}, \\
& \underline{z}^{\underline{k}}=z_{1}^{k_{1}} z_{2}^{k_{2}} \cdots z_{s}^{k_{s}}, \\
& \underline{A}=\left(A_{1}, A_{2}, \cdots, A_{s}\right), \\
& (\underline{A})=A_{1}+A_{2}+\cdots+A_{s}, \\
& \underline{B}=\left(B_{1}, B_{2}, \cdots, B_{s}\right), \\
& (\underline{B})=B_{1}+B_{2}+\cdots+B_{s}, \\
& (\underline{A+I})_{\underline{k}}=\left(A_{1}+I\right)_{k_{1}}\left(A_{2}+I\right)_{k_{2}} \cdots\left(A_{s}+I\right)_{k_{s}}, \\
& (\underline{B+I})_{\underline{k}}=\left(B_{1}+I\right)_{k_{1}}\left(B_{2}+I\right)_{k_{2}} \cdots\left(B_{s}+I\right)_{k_{s}}
\end{aligned}
$$

and

$$
\underline{J_{A, B}}=\left(J_{A_{1}, B_{1}}, J_{A_{2}, B_{2}}, \cdots, J_{A_{S}, B_{S}}\right) .
$$

Suppose that order N. Construct the function

$$
\begin{aligned}
\underline{J_{A, B}}(\underline{z})= & \left(\frac{\underline{z}}{3}\right)^{\underline{A+B}} \Gamma^{-1}(\underline{A+I}) \Gamma^{-1}(\underline{B+I}) \\
& \times \underline{{ }_{0} F_{2}}\left(-,-; \underline{A+I}, \underline{B+I} ; \underline{\underline{z^{3}}}\right) \\
= & \sum_{\underline{k}=0}^{\infty} U_{\underline{A+B+3 k I}} z^{\underline{A+B+3 k L}},
\end{aligned}
$$

where

$$
U_{\underline{A+B+3 k I}}=\frac{(-1)^{\underline{k}} \Gamma^{-1}(\underline{A+(k+1) I}) \Gamma^{-1} \underline{(B+(k+1) I)}}{3^{\underline{A+B+3 k I}} \underline{\underline{k}} .} .
$$

$$
\begin{aligned}
J_{A_{i}, B_{i}}\left(z_{i}\right)= & \left(\frac{z_{i}}{3}\right)^{A_{i}+B_{i}} \Gamma^{-1}\left(A_{i}+I\right) \Gamma^{-1}\left(B_{i}+I\right) \\
& \times{ }_{0} F_{2}^{i}\left(-,-; A_{i}+I, B_{i}+I ;-\frac{z_{i}^{3}}{27}\right) ; i=1,2, \cdots, s
\end{aligned}
$$

are $s$ Humbert matrix function with square complex matrices $A_{1}, A_{2}, \cdots, A_{s}$ and $B_{1}, B_{2}, \cdots, B_{s}$ of the same

This function, will be called the composite Humbert $z_{1}, z_{2}, \cdots, z_{s}$. Now we prove that the matrix power series (2.3) convergence for all $\underline{z}^{3} \neq 0$. Using the ratio test, we 
Note that if $k+1$ is large enough so that $k+1>\|A\|$, then by perturbation lemma, [13], we can write

$$
\begin{aligned}
& \left\|(A+(k+1) I)^{-1}\right\|=\left\|\frac{1}{k+1}\left(\frac{A}{k+1}+I\right)^{-1}\right\| \\
& =\frac{1}{k+1}\left\|\left(\frac{A}{k+1}+I\right)^{-1}\right\| \leq \frac{1}{k+1-\|A\|}
\end{aligned}
$$

hence

$$
\begin{aligned}
\frac{1}{R}= & \limsup _{(\underline{k}) \rightarrow \infty}\left[\frac{1}{k_{1}+1 . . k_{s}+1\left(k_{1}+1-\left\|A_{1}\right\|\right) . .}\right. \\
& \times \frac{1}{. .\left(k_{s}+1-\left\|A_{s}\right\|\right)\left(k_{1}+1-\left\|B_{1}\right\|\right) . .} \\
& \left.\times \frac{\left|\underline{z}^{3}\right|}{. .\left(k_{s}+1-\left\|B_{s}\right\|\right)}\right] .
\end{aligned}
$$

For positive numbers $\mu_{i}$ and positive integer $k$, we can write

$$
k_{i}=\mu_{i} k, \quad i=1,2,2 \cdots s .
$$

Substitute from (2.6) into (2.5) one gets

$$
\frac{1}{R}=\limsup _{(\underline{k}) \rightarrow \infty}\left\|\frac{U_{A+B+3(k+1) I} \underline{z}^{\underline{A+B+3(k+1) I}}}{U_{\underline{A+B+3 k I}} \underline{Z}^{A+B+3 k I}}\right\|=0 .
$$

Thus, the power series (2.3) is absolutely convergent for all $\left|\underline{z}^{3}\right|<\infty$.

\section{Integral Form of the Composite Humbert Matrix \\ Function}

To get an integral form for the composite Humbert matrix function, we use the following formulas (see [10, 12 , p. 115 , No. (5.10.5)]).

$$
\begin{aligned}
& \Gamma^{-1}\left(A_{1}+\left(k_{1}+1\right) I\right)=\frac{1}{2 \pi i} \int_{C_{1}} \exp \left(r_{1}\right) r_{1}^{-\left(A_{1}+\left(k_{1}+1\right) I\right)} \mathrm{d} r_{1}, \\
& \Gamma^{-1}\left(A_{2}+\left(k_{2}+1\right) I\right)=\frac{1}{2 \pi i} \int_{C_{2}} \exp \left(r_{2}\right) r_{2}^{-\left(A_{2}+\left(k_{2}+1\right) I\right)} \mathrm{d} r_{2}, \\
& \vdots \\
& \Gamma^{-1}\left(A_{s}+\left(k_{s}+1\right) I\right)=\frac{1}{2 \pi i} \int_{C_{s}} \exp \left(r_{s}\right) r_{s}^{-\left(A_{s}+\left(k_{s}+1\right) I\right)} \mathrm{d} r_{s},
\end{aligned}
$$

and

$$
\begin{aligned}
& \Gamma^{-1}\left(B_{1}+\left(k_{1}+1\right) I\right)=\frac{1}{2 \pi i} \int_{C_{1}^{\prime}} \exp \left(t_{1}\right) t_{1}^{-\left(B_{1}+\left(k_{1}+1\right) I\right)} \mathrm{d} t_{1}, \\
& \Gamma^{-1}\left(B_{2}+\left(k_{2}+1\right) I\right)=\frac{1}{2 \pi i} \int_{C_{2}^{\prime}} \exp \left(t_{2}\right) t_{2}^{-\left(B_{2}+\left(k_{2}+1\right) I\right)} \mathrm{d} t_{2}, \\
& \vdots \\
& \Gamma^{-1}\left(B_{s}+\left(k_{s}+1\right) I\right)=\frac{1}{2 \pi i} \int_{C_{s}^{\prime}} \exp \left(t_{s}\right) t_{s}^{-\left(B_{s}+\left(k_{s}+1\right) I\right)} \mathrm{d} t_{s} .
\end{aligned}
$$

From (2.7) and (2.8) into the series expression of the composite Humbert matrix function given in (2.3), it follows that

$$
\begin{aligned}
\underline{J_{A, B}}(\underline{z})= & \sum_{\underline{k}=0}^{\infty} \frac{(-1)^{\underline{k}}\left(\frac{z}{\underline{z}}\right)^{\underline{A+B+3 k I}}}{\underline{k}(2 \pi i)^{2 s}} \int_{C_{1}} \exp \left(r_{1}\right) r_{1}^{-\left(A_{1}+\left(k_{1}+1\right) I\right)} \mathrm{d} r_{1} . . \\
& . . \int_{C_{s}} \exp \left(r_{s}\right) r_{1}^{-\left(A_{s}+\left(k_{1}+1\right) I\right)} \mathrm{d} r_{s} \\
& \int_{C_{1}} \exp \left(t_{1}\right) t_{1}^{-\left(B_{1}+\left(k_{1}+1\right) I\right)} \mathrm{d} t_{1} . . \\
& . . \int_{C_{s}^{\prime}} \exp \left(t_{s}\right) t_{s}^{-\left(B_{s}+\left(k_{s}+1\right) I\right)} \mathrm{d} t_{s},
\end{aligned}
$$
interchanging the order of the integral and summation,

$$
\begin{aligned}
J_{A, B}(\underline{z})= & \frac{(\underline{\underline{z}})^{\underline{A+B}}}{(2 \pi i)^{2 s}} \int_{C_{1}} \exp \left(r_{1}\right) r_{1}^{-\left(A_{1}+I\right)} \mathrm{d} r_{1} . . \\
& . \int_{C_{s}} \exp \left(r_{s}\right) r_{1}^{-\left(A_{s}+I\right)} \mathrm{d} r_{s} \\
& \int_{C_{1}^{\prime}} \exp \left(t_{1}\right) t_{1}^{-\left(B_{1}+I\right)} \mathrm{d} t_{1} . \\
& . \int_{C_{s}^{\prime}} \exp \left(t_{s}\right) t_{s}^{-\left(B_{s}+I\right)} \mathrm{d} t_{s} \sum_{\underline{k}=0}^{\infty} \frac{(-1)^{\underline{k}}\left(\frac{\underline{z}^{3}}{27 \underline{r} \underline{t}}\right)^{\underline{k}}}{\underline{k} !}
\end{aligned}
$$

thus,

$$
\begin{aligned}
\underline{J_{A, B}}(\underline{z})= & \frac{(\underline{\underline{z}})^{\underline{A+B}}}{(2 \pi i)^{2 s}}\left(\int_{C_{1}} \cdots \int_{C_{s}} \int_{C_{1}^{\prime}} \cdots \int_{C_{s}^{\prime}} \exp \left(r+t-\frac{z^{3}}{27 r t}\right)\right. \\
& \times \underline{r}^{-(\underline{A+I})} \underline{t}^{-(\underline{B+I})}\left(\mathrm{d} r_{1} \cdots \mathrm{d} r_{s} \mathrm{~d} t_{1} \cdots \mathrm{d} t_{s}\right),
\end{aligned}
$$

where

$$
\begin{gathered}
\underline{r}=\left(r_{1}, r_{2}, \cdots, r_{s}\right), \\
\underline{t}=\left(t_{1}, t_{2}, \cdots, t_{s}\right), \\
(\underline{r})=r_{1}+r_{2}+\cdots+r_{s}, \\
(\underline{t})=t_{1}+t_{2}+\cdots+t_{s}, \\
(\underline{A+I})=A_{1}+I \cdots A_{s}+I, \\
(\underline{B+I})=B_{1}+I \cdots B_{s}+I
\end{gathered}
$$

and

$$
\underline{\left(\frac{z^{3}}{27 r t}\right)}=\frac{z_{1}^{3}}{27 r_{1} t_{1}}+\frac{z_{2}^{3}}{27 r_{2} t_{2}}+\ldots+\frac{z_{s}^{3}}{27 r_{s} t_{s}} .
$$

Therefore, the following result has been established.

Theorem 2.1 Let $\underline{A}$ and $\underline{B}$ be matrices in $C^{N \times N}$. Then the composit Humbert matrix function for several 
complex variables $z_{1}, z_{2}, \cdots, z_{s}$ satisfies integral in (2.11).

\section{Humbert Kummer Matrix Function}

In this section, we will deduce a new matrix function that is a mixed from Humbert matrix function is in the form

$$
\begin{aligned}
J_{A, n I}(z) & =\left(\frac{z}{3}\right)^{A+n I} \Gamma^{-1}(A+I) \Gamma^{-1}((n+1) I) \\
& \times{ }_{0} F_{2}\left(-,-; A+I,(n+1) I ;-\frac{z^{3}}{27}\right)
\end{aligned}
$$

and Kummer matrix function is in the form

$$
K(B, C ;-; z)=\sum_{n=0}^{\infty} \frac{(B)_{n}(C)_{n}}{n !}\left(\frac{z}{3}\right)^{-n}
$$

this function given the following

$$
\begin{aligned}
& M(A, B, C ; z)=\sum_{n=0}^{\infty} \frac{(B)_{n}(C)_{n}}{n !}\left(\frac{z}{3}\right)^{-n} J_{A, n I}(z) \\
& =\left(\frac{z}{3}\right)^{A} \sum_{n=0}^{\infty} \frac{(B)_{n}(C)_{n}}{n !} \\
& \times \sum_{k=0}^{\infty} \frac{(-1)^{k}\left[((k+1) I)_{n}\right]^{-1} \Gamma^{-1}(A+(k+1) I)\left(\frac{z}{3}\right)^{3 k}}{(k !)^{2}(k+1)_{n}} \\
& =\left(\frac{z}{3}\right)^{A} \sum_{k=0}^{\infty} \frac{(-1)^{k} \Gamma^{-1}(A+(k+1) I)\left(\frac{z}{3}\right)^{3 k}}{(k !)^{2}} \\
& \times \sum_{n=0}^{\infty} \frac{(B)_{n}(C)_{n}\left[((k+1) I)_{n}\right]^{-1}}{n !}
\end{aligned}
$$

by Theorem 2.1 in [9], we know that

$$
\begin{aligned}
& \sum_{n=0}^{\infty} \frac{(B)_{n}(C)_{n}\left[((k+1) I)_{n}\right]^{-1}}{n !} \\
& ={ }_{2} F_{1}(B, C ;(k+1) I ; 1) \\
& =\Gamma(k+1) \Gamma((k+1) I-B-C) \\
& \times \Gamma^{-1}((k+1) I-B) \Gamma^{-1}((k+1) I-C), \\
& \gamma((k+1) I-B-C)>0 \text {. Hence } \\
& M(A, B, C ; z)=\sum_{n=0}^{\infty} \frac{(B)_{n}(C)_{n}}{n !}\left(\frac{z}{3}\right)^{-n} J_{A, n I}(z) \\
& =\left(\frac{z}{3}\right)^{A} \Gamma(I-B-C) \Gamma^{-1}(A+I) \Gamma^{-1}(I-B) \Gamma^{-1}(I-C) \\
& \times{ }_{1} F_{3}\left(I-B-C ; A+I, I-B, I-C ;-\frac{z^{3}}{27}\right), \\
& =\sum_{k=0}^{\infty} U_{A+3 k I} z^{A+3 k I} .
\end{aligned}
$$

Provided that $\gamma(B+C)>0$. Where $A, B$ and $C$ are matrices in $C^{N \times N}$ such that $A+(k+1) I$, $(k+1) I-C$ and $(k+1) I-B$ are invertible for every integer $k \geq-1$. This function, will be called the Humber Kummer matrix function of complex variable $z$. For simplicity, we can write the Humber Kummer matrix function in the form HKMF.

We define the radius of regularity of the function $M(A, B, C ; z)$ in the form

$$
\begin{aligned}
\frac{1}{R} & =\limsup _{k \rightarrow \infty} \|\left(U_{A+3 k I}\right)^{\frac{1}{A+3 k I} \|} \\
& \leq \limsup _{k \rightarrow \infty}\left(\frac{\left\|k^{(I-(B+C))} k^{-(A+I)} k^{-(I-B)} k^{-(I-C)}\right\|}{((k-1) !)^{2} k !}\right)^{\frac{1}{A+3 k I}} \\
& \leq \limsup _{k \rightarrow \infty}\left(\frac{\left\|k^{-A}\right\|}{(k !)^{3}}\right)^{\frac{1}{A+3 k l}}=0 .
\end{aligned}
$$

This means that the the HKMF is an entire function.

\section{Integral Expressions of Humbert Kummer Matrix Function}

In this section, we provide integral expressions of HKMF. Suppose that $(I-(B+C))$ and $(A+I)$ are matrices in $C^{N \times N}$ such that

$(I-(B+C))(A+I)=(A+I)(I-(B+C))$,

$(I-(B+C)),(A+I)$ and $(A+B+C)$ are positive stable.

By (1.5) and (3.6) one gets

$$
\begin{aligned}
&(I-(B+C))_{k}\left[(A+I)_{k}\right]^{-1} \\
&= \Gamma^{-1}(I-(B+C)) \Gamma((1+k) I-(B+C)) \\
& \Gamma(A+I) \Gamma^{-1}(A+(k+1) I) \\
&= \Gamma^{-1}(I-(B+C)) \Gamma(A+I) \Gamma^{-1}(A+B+C) \\
& \Gamma((1+k) I-(B+C)) \Gamma(A+B+C) \Gamma^{-1}(A+(k+1) I) .
\end{aligned}
$$

By lemma 2 of [19] and (3.7), we see that

$\Gamma((1+k) I-(B+C)) \Gamma(A+B+C) \Gamma^{-1}(A+(k+1) I)$

$=\int_{0}^{1} t^{k-(B+C)}(1-t)^{A+B+C-I} \mathrm{~d} t$.

From relation (3.7) and (3.8), we get

$$
\begin{aligned}
& (I-(B+C))_{k}\left[(A+I)_{k}\right]^{-1} \\
& =\Gamma^{-1}(I-(B+C)) \Gamma(A+I) \Gamma^{-1}(A+B+C) \\
& \times \int_{0}^{1} t^{k-(B+C)}(1-t)^{A+B+C-I} \mathrm{~d} t .
\end{aligned}
$$


Hence, for $|z|<\infty$, we can write

$$
\begin{aligned}
& M(A, B, C ; z) \\
= & \left(\frac{z}{3}\right)^{A} \Gamma^{-1}(I-B) \Gamma^{-1}(I-C) \Gamma^{-1}(A+B+C) \\
& \sum_{k=0}^{\infty} \int_{0}^{1} t^{k-(B+C)}(1-t)^{A+B+C-I} \mathrm{~d} t \\
\times & \frac{(-1)^{k}\left[(I-B)_{k}\right]^{-1}\left[(I-C)_{k}\right]^{-1}\left(\frac{z}{3}\right)^{3 k}}{k !} \\
= & \left(\frac{z}{3}\right)^{A} \Gamma^{-1}(I-B) \Gamma^{-1}(I-C) \Gamma^{-1}(A+B+C) \\
& \int_{0}^{1} t^{-(B+C)}(1-t)^{A+B+C-I} \mathrm{~d} t \\
\times & \sum_{k=0}^{\infty} \frac{(-1)^{k}\left[(I-B)_{k}\right]^{-1}\left[(I-C)_{k}\right]^{-1}\left(\frac{z \sqrt[3]{t}}{3}\right)^{3 k}}{k !} \\
= & \left(\frac{z}{3}\right)^{A} \Gamma^{-1}(I-B) \Gamma^{-1}(I-C) \Gamma^{-1}(A+B+C) \\
& \int_{0}^{1} t^{-(B+C)}(1-t)^{A+B+C-I} \\
\times & \times{ }_{0} F_{2}\left(-; I-B, I-C ;-\left(\frac{z \sqrt[3]{t}}{3}\right)^{3}\right) \mathrm{d} t .
\end{aligned}
$$

Summarizing, the following result has been established:

Theorem 3.1 Let $A, B$ and $C$ be matrices in $C^{N \times N}$ such that $\gamma(A)>1$,

$(I-(B+C))(A+I)=(A+I)(I-(B+C))$ and
$(I-(B+C)),(A+I),(A+B+C)$ are positive stable. Then for $|z|<\infty$ it follows that

$$
\begin{aligned}
M(A, B, C ; z) & \left(\frac{z}{3}\right)^{A} \Gamma^{-1}(I-B) \Gamma^{-1}(I-C) \Gamma^{-1}(A+B+C) \\
& \left(\int_{0}^{1} t^{-(B+C)}(1-t)^{A+B+C-I}\right. \\
\times & \left.{ }_{0} F_{2}\left(-; I-B, I-C ;-\left(\frac{z \sqrt[3]{t}}{3}\right)^{3}\right) \mathrm{d} t\right)
\end{aligned}
$$

Another integral representation of HKMF can be established starting from the formula in (2.7), we find that

$$
\Gamma^{-1}(A+(k+1) I)=\frac{1}{2 \pi i} \int_{C} \exp (r) r^{-(A+(k+1) I)} \mathrm{d} r
$$

and substituting the above expression into the series expression of the HKMF given in (3.4), it follows that

$$
\begin{aligned}
& M(A, B, C ; z) \\
& =\left(\frac{z}{3}\right)^{A} \Gamma(I-B-C) \Gamma^{-1}(I-B) \Gamma^{-1}(I-C) \\
& \sum_{k=0}^{\infty} \frac{(-1)^{k}(I-(B+C))_{k}\left[(I-B)_{k}\right]^{-1}\left[(I-C)_{k}\right]^{-1}\left(\frac{z}{3}\right)^{3 k}}{k !} \\
& \times \frac{1}{2 \pi i} \int_{C} \exp (r) r^{-(A+(k+1) I)} \mathrm{d} r,
\end{aligned}
$$

interchanging the order of the integral and summation,

$$
\begin{aligned}
M(A, B, C ; z)= & \frac{1}{2 \pi i}\left(\frac{z}{3}\right)^{A} \Gamma(I-B-C) \Gamma^{-1}(I-B) \Gamma^{-1}(I-C) \int_{C} \exp (r) r^{-(A+I)} \mathrm{d} r \\
& \sum_{k=0}^{\infty} \frac{(-1)^{k}(I-(B+C))_{k}\left[(I-B)_{k}\right]^{-1}\left[(I-C)_{k}\right]^{-1}\left(\frac{z}{3 \sqrt[3]{r}}\right)^{3 k}}{k !},
\end{aligned}
$$

i.e.,

$$
\begin{aligned}
M= & \frac{1}{2 \pi \mathrm{i}}\left(\frac{z}{3}\right)^{A} \Gamma(I-B-C) \Gamma^{-1}(I-B) \Gamma^{-1}(I-C) \\
& \left(\int_{C} \exp (r) r^{-(A+I)}{ }_{1} F_{2}\left(I-B-C ; I-B, I-C ;-\left(\frac{Z}{3 \sqrt[3]{r}}\right)^{3}\right) \mathrm{d} r\right) .
\end{aligned}
$$

Therefore, we obtain the following theorem:

Theorem 3.2 Let $A, B$ and $C$ be matrices in
$C^{N \times N}$ such that $\gamma(B+C)<1$. Then for $|z|<\infty$, expression (3.11) hold true. 


\section{Composite HKMF}

Let

$$
\begin{aligned}
& M_{i}\left(A_{i}, B_{i}, C_{i} ; z_{i}\right) \\
& =\left(\frac{z_{i}}{3}\right)^{A_{i}} \Gamma\left(I-B_{i}-C_{i}\right) \Gamma^{-1}\left(A_{i}+I\right) \Gamma^{-1}\left(I-B_{i}\right) \\
& \Gamma^{-1}\left(I-C_{i}\right){ }_{1} F_{3}^{i}\left(I-B_{i}-C_{i} ; A_{i}+I, I-B_{i}, I-C_{i} ;-\frac{z_{i}^{3}}{27}\right),
\end{aligned}
$$

are composite Humbert Kummer matrix functions with square complex matrices $A_{i}, B_{i}$ and $C_{i}$ of the same order $N$, provided that $\gamma\left(B_{i}+C_{i}\right)<1$.

Construct the composite Humbert Kummer matrix functions of these functions for any mode of arrangement, we put

$$
\begin{aligned}
& \underline{M}(\underline{A}, \underline{B}, \underline{C} ; \underline{z}) \\
& =\left(\frac{\underline{Z}}{3}\right)^{\underline{A}} \Gamma \underline{(I-(B+C)) \Gamma^{-1}} \underline{(A+I)} \Gamma^{-1} \underline{(I-B)} \Gamma^{-1} \underline{(I-C)}
\end{aligned}
$$

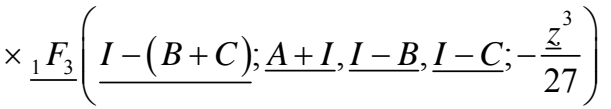

$$
\begin{aligned}
& ={ }_{1} F_{3}\left(I-(B+C) ; \underline{A+I}, \underline{I-B}, \underline{I-C} ;-\frac{\underline{\underline{z}}^{3}}{27}\right)
\end{aligned}
$$

where

$$
\begin{aligned}
& \underline{B}=\left(B_{1}, B_{2}, \cdots, B_{s}\right), \\
& \underline{C}=\left(C_{1}, C_{2}, \cdots, C_{s}\right), \\
& (\underline{I-B-C})_{\underline{k}}=\left(I-B_{1}-C_{1}\right)_{k_{1}} \cdots\left(I-B_{s}-C_{s}\right)_{k_{s}}, \\
& (\underline{I-B})_{\underline{k}}=\left(I-B_{1}\right)_{k_{1}}\left(I-B_{2}\right)_{k_{2}} \cdots\left(I-B_{s}\right)_{k_{s}}, \\
& (\underline{I-C})_{\underline{k}}=\left(I-C_{1}\right)_{k_{1}}\left(I-C_{2}\right)_{k_{2}} \cdots\left(I-C_{s}\right)_{k_{s}},
\end{aligned}
$$

and

$$
\underline{M}=\left(M_{1}, M_{2}, \cdots, M_{s}\right) .
$$

Then $M$ is the composite Humbert Kummer matrix functions. Now we calculate the radius of convergence of this function as follows

$$
\begin{aligned}
\frac{1}{R} & =\limsup _{k \rightarrow \infty}\left\|\left(\frac{U_{\underline{A}+3 k I}}{\sigma_{\underline{k}}}\right)^{\frac{1}{A+3 k I}}\right\| \\
& \leq \limsup _{\langle\underline{k}\rangle \rightarrow \infty}\left(\frac{\left\|k_{1}^{-A_{1}}\right\|\left\|k_{2}^{-A_{2}}\right\| \cdots\left\|k_{s}^{-A_{s}}\right\|}{\left(\underline{k_{1}} !\right)^{3}\left(\underline{k_{2}} !\right)^{3} \cdots\left(\underline{k_{s} !}\right)^{3}}\right)^{\frac{1}{A+3 k l}}
\end{aligned}
$$

where

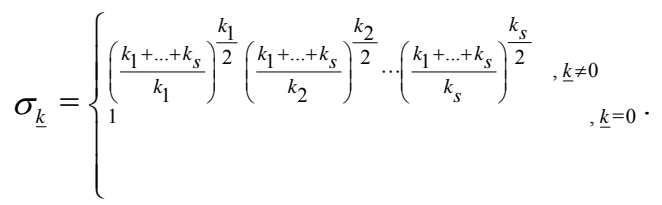

as above putting $k_{i}=\mu_{i} k$ and using relation (1.9) and the following relation

$$
\begin{aligned}
\sum_{j=0}^{r-1} \frac{\left(\|A\| r^{\frac{1}{2}} \ln (k)^{j}\right.}{j !} & \leq\left(r \ln (k)^{r-1} \sum_{j=0}^{r-1} \frac{(\|A\|)^{j}}{j !}\right. \\
& \leq\left(r \ln (k)^{r-1} \sum_{j=0}^{\infty} \frac{(\|A\|)^{j}}{j !}\right. \\
& =(r \ln k)^{r-1} \mathrm{e}^{\|A\|},
\end{aligned}
$$

we get

$$
\frac{1}{R}=\limsup _{k \rightarrow \infty}\left\|\left(\frac{U_{\underline{A+3 k I}}}{\sigma_{\underline{k}}}\right)^{\frac{1}{A+3 k I}}\right\|=0 .
$$

Then the composite Humbert Kummer matrix functions is an entire function.

\section{References}

[1] A. G. Constantine and R. J. Mairhead, "Partial Differential Equations for Hypergeometric Function of Two Argument Matrix," Journal of Multivariate Analysis, Vol. 2, No. 3, 1972, pp. 332-338. doi:10.1016/0047-259X(72)90020-6

[2] A. T. James, "Special Functions of Matrix and Single Argument in Statistics in Theory and Application of Special Functions," Academic Press, New York, 1975.

[3] A. M. Mathai, "A Handbook of Generalized Special Functions for Statistical and Physical Sciences," Oxford University Press, Oxford, 1993.

[4] A. M. Mathai, "Jacobians of Matrix Transformations and Functions of Matrix Argument," World Scientific Publishing, New York, 1997.

[5] L. Jodar and E. Defez, "A Connection between Lagurre's and Hermite's Matrix Polynomials," Applied Mathematics Letters, Vol. 11, 1998, pp. 13-17.

[6] E. Defez and L. Jódar, "Chebyshev Matrix Polynomails and Second Order Matrix Differential Equations," Utilitas Mathematics, Vol. 61, 2002, pp. 107-123.

[7] E. Defez and L. Jódar, "Some Applications of the Hermite Matrix Polynomials Series Expansions," Journal of Computational and Applied Mathematics, Vol. 99, No. 1-2, 1998, pp. 105-117. doi:10.1016/S0377-0427(98)00149-6

[8] J. Sastre and L. Jódar, "Asymptotics of the Modified Bessel and Incomplete Gamma Matrix Functions," Ap- 
plied Mathematics Letters, Vol. 16, No. 6, 2003, pp. 815820. doi:10.1016/S0893-9659(03)90001-2

[9] L. Jódar, R. Company and E. Navarro, "Bessel Matrix Functions: Explict Solution of Coupled Bessel Type Equations," Utilitas Mathematics, Vol. 46, 1994, pp. 129-141.

[10] Z. M. G. Kishka, A. Shehata and M. Abul-Dahab, "On Humbert Matrix Function,” Applied Mathematics Letters, Article in Press.

[11] S. Z. Rida, M. Abul-Dahab, M. A. Saleem and M. T. Mohammed, "On Humbert Matrix Function $\Psi_{1}\left(A, B ; C, C^{\prime} ; z, w\right)$ of Two Complex Variables under Differential Operator," International Journal of Industrial Mathematics, Vol. 32, 2010, pp. 167-179.

[12] N. N. Lebedev, "Special Functions and Their Applications," Dover Publications Inc., New York, 1972.

[13] L. Y. Luke, "The Special Functions and Their Approximations," Vol. 2, Academic Press, New York, 1969.

[14] H. M. Srivastava and P. W. Karlsson, "Multiple Gaussian Hypergeometric Series," Ellis Horwood, Chichester, 1985.
[15] M. S. Metwally, “On p-Kummers Matrix Function of Complex Variable under Differential Operators and Their Properties," Southeast Asian Bulletin of Mathematics, Vol. 35, 2011, pp. 1-16.

[16] A. Shehata, "A Study of Some Special Functions and Polynomials of Complex Variables," Ph.D. Thesis, Assiut University, Assiut, 2009.

[17] L. Jódar and J. C. Cortés, "On the Hypergeometric Matrix Function," Journal of Computational and Applied Mathematics, Vol. 99, No. 1-2, 1998, pp. 205-217. doi:10.1016/S0377-0427(98)00158-7

[18] G. Golub and C. F. Van Loan, "Matrix Computations," The Johns Hopkins University Press, Baltimore, 1989.

[19] L. Jódar and J. C. Cortés, "Some Properties of Gamma and Beta Matrix Functions," Applied Mathematics Letters, Vol. 11, No. 1, 1998, pp. 89-93. doi:10.1016/S0893-9659(97)00139-0

[20] K. A. M. Sayyed, M. S. Metwally and M. T. Mohamed, "Certain Hypergeometric Matrix Function, "Scientiae Mathematicae Japonicae, Vol. 69, No. 3, 2009, pp. 315321. 